\title{
Welfare losses under Cournot competition ${ }^{\text {th }}$
}

\author{
Luis C. Corchón \\ Departamento de Economía, Universidad Carlos III, c/ Madrid 126, Getafe, Madrid 28903, Spain
}

Received 19 July 2007; received in revised form 30 October 2007; accepted 31 October 2007

\begin{abstract}
In a market for a homogeneous good where firms are identical, compete in quantities and produce with constant returns, the percentage of welfare losses (PWL) is small with as few as five competitors for a class of demand functions which includes linear and isoelastic cases. We study markets with positive fixed costs and asymmetric firms. We provide exact formulae of PWL and robust constructions of markets were PWL is close to one in these two cases. We show that the market structure that maximizes PWL is either monopoly or dominant firm, depending on demand. Finally we prove that PWL is minimized when all firms are identical, a clear indication that the assumption of identical firms biases the estimation of PWL downwards.
\end{abstract}

(C) 2007 Elsevier B.V. All rights reserved.

Keywords: Welfare losses; Asymmetric firms; Fixed costs

\section{Introduction}

In his classical contribution Cournot (1838, Chapter 8) established that when the number of firms in a market tends to infinity, oligopolistic equilibrium tends to perfect competition. As a corollary, Welfare Losses (WL), measured as the difference between social welfare in the optimal and the equilibrium allocation, tend to zero. But, what happens when the number of firms is finite? Is perfect competition a good approximation or, on the

\footnotetext{
it This paper was presented in the universities of Murcia, Pablo de Olavide (Sevilla) and Carlos III and in the Social Science Research Center (WZB) in Berlin. I am grateful to the audiences there, to C. Beviá, N. Fabra, J. Gans, M. González-Maestre, C. Gozález-Pimienta, J. Jaumandreu, J. López-Cuñat, C. Litan, F. Marcos, M. Moller, D. Moreno, C. Ponce and G. Zudenkova and to an anonymous referee for helpful comments and to the Spanish Ministry of Education for financial support under grant SEJ2005-06167.

E-mail address: 1corchon@eco.uc3m.es.
}

contrary are WL significant? (see Hotelling (1938) and 26 Yarrow (1985) for an early treatment of this problem). 27

As a first cut to the problem, assume that all firms are 28 identical and costs and demand are linear. It is easily 29 calculated that the percentage of WL under Cournot 30 competition, denoted by PWL, is $1 /(1+n)^{2}$ where $n$ is 31 the number of firms. Thus, despite the fact that monop- 32 oly and duopoly entail large PWL this magnitude goes 33 to zero pretty quickly: a market composed by 7 identical 34 firms ("the seven sisters") produces a PWL of $1.56 \% 35$ only. ${ }^{1}$ This poses a serious question: were WL system- 36 atically small a simple equilibrium concept like perfect 37 competition may be preferable as a description of mar- 38 kets unless an additional argument is made in favor of 39

\footnotetext{
${ }^{1}$ This formula shows that once linearity is assumed, as done implicitly by Harberger (1954), WL seldom goes up to big numbers except if the number of firms is very small. A list of other empirical papers measuring WL in oligopoly can be found in Tullock (2003) p. 2.
} 
the Cournot model (e.g. that the distribution of the surplus in Cournot and perfect competition is very different). Moreover, the motivation for public policies dealing with efficiency is lost under small WL.

Let us first comment on papers that deal with our problem. McHardy (2000) studies a model with quadratic demand and presents numerical calculations. He finds that WL can be up to $30 \%$ larger than those in the linear model, which is encouraging but still does not solve the problem. Anderson and Renault (2003) calculate PWL under the assumptions made above except that they assume an inverse demand function of the form $p=A-b x^{\alpha},(x$ is aggregate output and $p$ market price). ${ }^{2}$ They do not study if PWL differs substantially from those in the linear model. Johari and Tsitsiklis (2005) show that if firms are identical, average costs are not increasing and the inverse demand function is concave, PWL is bounded above by $1 /(2 n+1)$, which is still not very large because a market with seven firms achieves, at least, $93.33 \%$ of maximum welfare.

Our paper is a quest for markets where oligopoly produces large WL. Specifically, the purpose of our paper is twofold: to provide workable formulae for PWL which depend, as far as possible, on magnitudes that are observable. ${ }^{3}$ And to use these formulae to construct markets where the Cournot equilibria yields large PWL. ${ }^{4}$

In Section 2 we consider the Baseline Model, which is that of Anderson and Renault. We might expect that for suitable values of $\alpha$, WL were much higher than those in the linear case. However, by using numerical methods we find that the maximum PWL obtained in this case is not very different from the one obtained in the linear case. Moreover, for some values of $\alpha$, PWL is arbitrarily small. Thus, the consideration of a more general class of demand functions does not bring significant WL associated with oligopoly, but on the contrary it adds to the suspicion that WL under oligopoly may be small. We then turn our attention to fixed costs and heterogeneous firms. ${ }^{5}$

\footnotetext{
${ }^{2}$ This form of demand generalizes both linear $(\alpha=1)$ and isoelastic (with elasticity of demand $1 / \alpha$ ) forms and allows for computation of equilibria.

${ }^{3}$ The parameter $\alpha$, which can be estimated but not observed, enters in the formula of PWL in Anderson and Renault (2003), so it is unavoidable in the more general set ups considered in this paper.

${ }^{4}$ Johari and Tsitsiklis (2005) offer an example of a market where PWL is arbitrarily close to one but in which the inverse demand function is not differentiable.

${ }^{5}$ Other attempts to find higher WL focus on issues outside market competition like "X-Inefficiency", Leibenstein (1966) and RentSeeking, Tullock (1967).
}

In Section 3 we consider free entry with a fixed cost. 80 We provide formulae for the maximal and the minimal 81 PWL where this magnitude depends on the number of 82 firms and $\alpha$. We show that when $\alpha$ and the fixed cost are 83 not observable, for any exogenously given observation 84 on market price, output, average variable cost and 85 number of firms, PWL can be chosen arbitrarily (Propo- 86 sition 1). In particular when $\alpha$ tends to infinity, PWL can 87 be chosen to be arbitrarily close to one. This result 88 implies that any given price-marginal costs margin, or 89 elasticity of demand, is compatible with any PWL. 90 When the fixed cost can be observed, the observed 91 variables must fulfill a condition which implies that 92 entry is blockaded. We show that any observation ful- 93 filling this condition is compatible with many - but not 94 all - PWL (Proposition 2).

In Section 4 we consider heterogeneous firms. We 96 provide a formula for PWL where this magnitude de- 97 pends (positively) on the share of the largest firm, 98 (negatively) on the Hirschman-Herfindahl concentra- 99 tion index, denoted by $H$, and on $\alpha$. We find that there 100 are markets with a large number of firms where PWL is 101 close to one whereas $H$ is close to zero (Proposition 3). 102 This shows that $H$ is not a reliable measure of WL. ${ }^{6} 103$ More importantly, it implies that the concept of a large 104 economy must be taken with care because seemingly 105 innocuous departs from a model where all firms are 106 small and identical may have serious welfare conse- 107 quences. Next, we prove that the market structure that 108 maximizes PWL is a dominant firm when $\alpha>0$ and 109 monopoly when $\alpha<0$ (Proposition 4). Thus, monopoly, 110 the target of attacks of our profession from Adam Smith 111 on, is not necessarily the worst outcome in terms of WL. 112 Finally we prove that PWL is minimized when firms are 113 identical (Proposition 5). This shows that proper care of 114 the heterogeneity of firms is essential to obtain estimates 115 of PWL that are not biased towards small PWL. 116

Finally, in Section 5 we offer some thoughts about 117 our results. Our main conclusion is twofold. On the one 118 hand, the search for WL in actual markets should focus 119 on economies of scale and asymmetric firms, two facts 120 that are seldom considered in the applied literature. On 121 the other hand the Cournot model can easily produce 122 large WL. Other important points are the characteriza- 123 tion of the best and the worst possible market structures 124 from the welfare point of view when firms are different 125

\footnotetext{
${ }^{6}$ That social welfare is increasing in the marginal cost of small firms was first pointed out by Lahiri and Ono (1988). For a criticism of the idea that concentration is generally bad for social welfare see Daughety (1990), Farrell and Shapiro (1990) and Cable et al. (1994).
} 

vestment, $R \& D$, location, etc. The analysis of the impact of these variables on WL requires the consideration of games that are more complicated than those considered here and, consequently, they are left for future research.

\section{The Baseline Model}

There is a representative consumer with a utility function $U=A x-\frac{b x^{x+1}}{\alpha+1}-p x$ where $x$ is aggregate output, $p$ is the market price, $b \alpha>0$ and $\alpha>-1$. The maximization of utility generates an inverse demand function $p=A-b x^{\alpha}$. Notice that if $\alpha<0, b<0$, and $A=0$ we have an isoelastic function $p=-b x^{\alpha}$. The linear case occurs if $\alpha=1$.

There are $n$ identical firms each producing a single output denoted by $x_{i}, i=1, \ldots, n$. Thus $x \equiv \sum_{i=1}^{n} x_{i}$. Marginal cost is constant and denoted by $c$. Profits for firm $i$ are $\pi_{i} \equiv(p-c) x_{i}$. Defining $\alpha \equiv A-c$ we have that $\pi_{i} \equiv\left(a=b x^{\alpha}\right) x_{i}$. Assume $a b>0$ and $-A \alpha<c n$. These assumptions guarantee that output and market price are positive in equilibrium (see Eq. (2.1)).

If firms compete in the manner of Cournot, the first order condition of profit maximization yields $a-b x^{\alpha}-$ $b \alpha x^{\alpha-1} x_{i}=0$. It is easy to check that the second order condition holds and that equilibrium is symmetric. Thus Cournot equilibrium output and market price are

From Eq. (2.3), the percentage of WL denoted by 166 PWL is

$P W L \equiv \frac{W^{o}-W^{*}}{W^{o}}=1-\frac{n^{\frac{1}{\alpha}}(n+\alpha+1)}{(n+\alpha)^{\frac{\alpha+1}{\alpha}}} \equiv L(\alpha, n)$,

\footnotetext{
${ }^{7}$ Other points that have already been noticed in the literature are the importance of the functional form of demand and the failure of the $H$ index and the price-marginal cost ratio to capture WL.
}

$x^{*}=\left(\frac{a n}{b(n+\alpha)}\right)^{\frac{1}{\alpha}}$ and $p^{*}=\frac{\mathrm{A} \alpha+c n}{n+\alpha}$.

Social welfare, denoted by $W$, is the sum of industry profits and the utility of the representative consumer, i.e. $W=a x-b \frac{x^{\alpha+1}}{1+\alpha}$. The optimal aggregate output is found by maximizing $W$, namely

$x^{o}=\left(\frac{a}{b}\right)^{\frac{1}{\alpha}}$.

Social welfare in equilibrium and in the optimal allocation, are, respectively

$W^{*}=\frac{a^{\frac{\alpha+1}{\alpha}} n^{\frac{1}{\alpha}} \alpha(n+\alpha+1)}{b^{\frac{1}{\alpha}}(n+\alpha)^{\frac{\alpha+1}{\alpha}}(\alpha+1)} \quad$ and $\quad W^{o}=\frac{a^{\frac{\alpha+1}{\alpha}} \alpha}{b^{\frac{1}{\alpha}}(\alpha+1)}$. see Anderson and Renault (2003) p. 262. The following 169 properties of $L(\cdot, \cdot)$ are easily proved:

i) $\lim _{n \rightarrow \infty} L(\alpha, n)=0$.

ii) $\lim _{\alpha \rightarrow-1} L(\alpha, n)=0$.

iii) $\lim _{n \rightarrow \infty} L(\alpha, n)=0$.

iv) $L(\alpha, \cdot)$ decreases with $n$.

v) $\mathrm{L}(\cdot, n)$ is quasi-concave in $\alpha$.

i) is the usual property of large economies, as noticed 177 in the Introduction. The explanation of ii) is that when 178 $\alpha \rightarrow-1$, the market produces in the limit an infinity 179 amount of surplus, so the loss caused by oligopoly tends 180 to zero. iii) is caused by the fact that when $\alpha \rightarrow \infty, 181$ inverse demand is flat so firms cannot influence price 182 and optimal and equilibrium output are identical. ii) and/ 183 or iii) imply that there are markets where, for a given $n, 184$ PWL is as small as we wish, something that is im- 185 possible in the case of quadratic utility functions. 186 iv) shows that, when there are no technological is- 187 sues at stake, the more competition, the better. Finally 188 v) follows from the fact that Anderson and Renault 189 (2003) proved that $W^{\mathrm{o}} / W^{*}$ is quasi-concave on $\alpha$. So 190 $W^{*} / W^{\circ}$ is quasi-convex and $-W^{*} / W^{\mathrm{o}}$ is quasi-con- 191 cave, so it is $1-W^{*} / W^{\circ}$.

We now study PWL as a function of $\alpha$. Table 1 below 193 shows, for selected values of $n$, the maximum PWL, 194 denoted by $\overline{\mathrm{PWL}}$, and PWL when the demand function 195 is linear, denoted by PWLL (see Corchón (2006) for 196 details). Notice that iv) above guarantees that for $n 197$ larger than $10, \overline{\mathrm{PWL}}$ will be smaller than $2.2 \%$.

Notice that the general form of the utility function 199 does not help much to obtain significant WL. Given this 200 and that PWL can be much smaller than $\overline{\mathrm{PWL}}$ (i.e. when 201 $\alpha$ is close to -1 or to $\infty$ ) we conclude that the con- 202 sideration of a more general class of utility functions 203 alone is not helpful to finding significant WL.

\section{Fixed costs and free entry}

In this section we assume that in order to produce, 206 firms must incur a fixed cost, denoted by $k$, and that there 207 is an infinity number of potential firms. The number of 208 active firms in equilibrium is denoted by $n$. Given $n, 209$ output is determined as in the previous section. We 210 assume that the decision of entry is prior to the decision 211 
Table 1

t1.1

$\mathrm{t} 1.2$

$\mathrm{t} 1.3$

212

213
In order to have a formula, in which PWL depends on observable variables, we substitute $k$ for its upper and lower bounds in Eq. (3.1). It is clear that PWL is increasing on $k$. Thus, the maximal PWL, denoted by MA $(\alpha, n)$, occurs for the maximum value of $k$, namely

$\operatorname{MA}(\alpha, n) \equiv \frac{(n+\alpha)^{\frac{1+\alpha}{\alpha}}-n^{\frac{1}{\alpha}}(n+\alpha+1)+(n-1) n^{\frac{1-\alpha}{\alpha}}(\alpha+1)}{(n+\alpha)^{\frac{1+\alpha}{\alpha}}-\alpha^{\frac{1-\alpha}{\alpha}}(\alpha+1)}$.

\footnotetext{
${ }^{8}$ López-Cuñat (1999) has shown that, under conditions that are met here, the equilibrium considered in this paper is a subset of an equilibrium when both decisions are simultaneous (like in Novshek (1980) and Ushio (1983)).
}

Minimal PWL, denoted by $\operatorname{MI}(\alpha, n)$, occurs for the 240 minimum value of $k$, namely

$$
\operatorname{MI}(\alpha, n) \equiv \frac{(n+\alpha+1)^{\frac{1+\alpha}{\alpha}}-\frac{n^{\frac{1}{\alpha}(n+\alpha+1) \frac{1+2 \alpha}{+\alpha}}}{(n+\alpha)^{\frac{1+\alpha}{\alpha}}}+(n-1)(n+1)^{\frac{1-\alpha}{\alpha}}(\alpha+1)}{(n+\alpha+1)^{\frac{1+\alpha}{\alpha}}-(n+1)^{\frac{1-\alpha}{\alpha}}(\alpha+1)} \cdot 242
$$

We now state the properties of and $\operatorname{MA}(\cdot, \cdot)$ and 244 $\operatorname{MI}(\cdot, \cdot)$ that correspond to i)-iv) in the previous section. 245

i') $\lim _{n \rightarrow \infty} \mathrm{MI}(\alpha, n)=\lim _{n \rightarrow \infty} \operatorname{MA}(\alpha, n)=0$.

ii') $\lim _{\alpha \rightarrow-1} \operatorname{MI}(\alpha, n)=\lim _{\alpha \rightarrow-1} \operatorname{MA}(\alpha, n)=0 . \quad 247$ iii') $\lim _{\alpha \rightarrow \infty} \mathrm{MI}(\alpha, n)=\frac{n-1}{n}, \lim \alpha \rightarrow \infty \mathrm{MA}(\alpha, n)=1.248$ iv') Neither $\operatorname{MI}(\alpha, \cdot)$ nor $\operatorname{MA}(\alpha, \cdot)$ are monotonic on $n .249$

i') implies that $\lim _{k \rightarrow 0} \mathrm{PWL}=0$, since Eq. (3.1) im- 251 plies that when $k \rightarrow 0, n \rightarrow \infty$. Variations of this result 252 have been obtained by Dasgupta and Ushio (1981), 253 Fraysse and Moreaux (1981) and Guesnerie and Hart 254 (1985). i') and ii') are identical to i) and ii) in the previous 255 section. However iii') is very different from iii) because it 256 says that markets with very large $\alpha^{\prime} s$ could be very 257 inefficient. For large values of $\alpha$, the contrast between 258 monopoly and markets with a large number of firms is 259 striking: In the former it is possible to construct examples 260 where PWL is arbitrarily small and in the latter such 261 examples are not possible. This is due to the fact that 262 when $n$ is very large, there are large WL due to the 263 discrepancy between the equilibrium and the optimal 264 number of firms, which is one. Finally iv') is proved by 265 means of an example available under request. The reason 266 for this - apparently paradoxical - result is that $k$ changes 267 in order to maintain the free entry condition (3.1). $\quad 268$

We now show that, if $k$ and $\alpha$ are unknown, PWL is 269 arbitrary even if certain variables - like price, output, 270 marginal cost and number of firms - are observed and 271 we require that they correspond to the values in a 272 Cournot Equilibrium with free entry for some para- 273 meters defining demand and costs. To formalize this, we 274 say that a Market is a list of real numbers $(A, c, b, \alpha, k) 275$ such that $k>0,(A-c) \alpha>0, \alpha>-1, \alpha b>0,-A \alpha<c n 276$ and $\alpha(A-c)^{\frac{\alpha+1}{\alpha}}>k b^{\frac{1}{\alpha}}(\alpha+1)$. An Observation is a list 277 $\left(\mathscr{P}, \mathscr{R}_{i}, \mathscr{C}, \mathscr{N}\right)$ where $\mathscr{P}$ is market price, $\mathscr{R}_{i}$ is output of 278 firm $i, \mathscr{C}(<\mathscr{P})$ is the marginal cost and $\mathscr{N}$ is the number 279 of active firms. The last variable is a positive integer and 280 
the others are positive real numbers. We assume that $c$ is observable because under constant returns, the marginal cost equals the average variable cost which, in principle, can be observed (wages, raw materials, etc.). Now we have the following:

Proposition 1. Given an observation $\left(\mathscr{P}, \mathscr{R}_{i}, \mathscr{C}, \mathscr{N}\right)$, and a number such that $v=\operatorname{MA}(\hat{\alpha}, n), \hat{\alpha} \in(-1,0) \cup(0, \infty)$, there is a market $(\hat{A}, \mathscr{C}, \hat{b}, \hat{\alpha}, \hat{k})$ such that $\left(\mathscr{P}, \mathscr{R}_{i}, \mathscr{N}\right)$ is a Cournot equilibrium with free entry for this market (i.e. they fulfill Eqs. (2.1) and (3.1)), and $\mathrm{PWL}=v$.

Proof. For $k$ equal to the maximum value in Eq. (3.1), PWL is given by Eq. (3.5). Let $v$ and $\hat{\alpha}$ be such that $\operatorname{MA}(\hat{\alpha}, \mathscr{N})=v$. Now set

$\hat{A}=\frac{\mathscr{P}(\mathscr{N}+\hat{\alpha})-\mathscr{C} \mathscr{N}}{\hat{\alpha}}, \quad \hat{k}=\frac{\hat{\alpha}(\hat{A}-\mathscr{C})^{\frac{1+\hat{\alpha}}{\alpha}} \mathscr{N}^{\frac{1-\hat{\alpha}}{\hat{\alpha}}}}{\hat{b}^{\frac{1}{\hat{\alpha}}}(\mathscr{N}+\hat{\alpha})^{\frac{1+\hat{\alpha}}{\hat{\alpha}}}}$,

$\hat{b}=\frac{(\hat{A}-\mathscr{C}) \mathscr{N}}{\mathscr{N}^{\hat{\alpha}} \mathscr{R}_{i}^{\hat{\alpha}}(\mathscr{N}+\hat{\alpha})}$

This system can be solved easily because the first equation determines $\hat{A}$, the last equation determines $\hat{b}$ and with these values of $\hat{A}$ and $\hat{b}$ the remaining equation determines $\hat{k}$. By construction $\hat{A} \hat{\alpha}=\mathscr{P}(\mathscr{N}+\hat{\alpha})-\mathscr{C} \mathscr{N}$, so $(\hat{A}-\mathscr{C}) \hat{\alpha}=\mathscr{P}(\mathscr{N}+\hat{\alpha})-\mathscr{C}(\mathscr{N}+\hat{\alpha})>0$ Then, from the last equation $\hat{\alpha} \hat{b}>0$ and the remaining equation implies $\hat{k}>0$. Also $\hat{A} \hat{\alpha}+\mathscr{C} \cdot \mathscr{N}=\mathscr{P}(\hat{N}+\hat{\alpha})>0$. Finally we will show that $\hat{\alpha}(\hat{A}-\mathscr{C})^{\frac{\hat{\alpha}+1}{\hat{\alpha}}}>\hat{k} \hat{b}^{\frac{1}{\alpha}}(\hat{\alpha}+1)$. Given the definitions of the parameters, this inequality $\operatorname{reads}(\mathscr{N}+\hat{\alpha})^{\frac{1+\hat{\alpha}}{\hat{\alpha}}}-\mathscr{N}^{\frac{1-\hat{\alpha}}{\hat{\alpha}}}(\hat{\alpha}+1)>0$. Call $\Psi(\hat{\alpha}, \mathcal{N})$ the left hand side of the previous inequality and extend the function to allow $n$ to take real values. Notice that $\Psi(\hat{\alpha}, 1)=(\hat{\alpha}+1)\left((\hat{\alpha}+1)^{\frac{1}{\hat{\alpha}}}-1\right)>0$. Also $\lim _{n \rightarrow \infty} \Psi(\hat{\alpha}$, $\mathscr{N})=\infty$. Then, if $\Psi(\hat{\alpha}, \mathscr{N}) \leq 0$ there must be a value of $\mathscr{N}$ say $\overline{\mathscr{N}}$ for which $\frac{\partial \Psi(\hat{\alpha}, \bar{N})}{\partial \mathcal{N}}=0$ and $\Psi(\hat{\alpha}, \bar{N}) \leq 0$. The former is equivalent to $(\bar{N}+\hat{\alpha})^{\frac{1}{\alpha}} \bar{N}=\bar{N}^{\frac{1-\hat{\alpha}}{\alpha}}(1-\hat{\alpha})$. If $\hat{\alpha}=1$ this is impossible. If $\hat{\alpha} \neq 1$ plugging this equa-

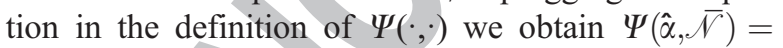
$(\bar{\Gamma}+\hat{\alpha})^{\frac{1}{\alpha}} \frac{\hat{\alpha}}{1-\hat{\alpha}}(-\hat{\alpha}+1-2 \bar{\gamma}) \neq 0$. Thus $\Psi(\hat{\alpha}, \bar{N})<0 \Leftrightarrow$ $\hat{\alpha} \in(0,1)$. However for $\hat{\alpha} \in(0,1),(\bar{N}+\hat{\alpha})^{\frac{1+\hat{\alpha}}{\alpha}} \geq \bar{N}^{\frac{1+\hat{\alpha}}{\hat{\alpha}}}$, so $\Psi(\hat{\alpha}, \overline{\mathcal{N}}) \geq \overline{\mathcal{N}}^{\frac{1}{\alpha}}\left(\overline{\mathcal{N}}-\frac{1+\hat{\alpha}}{\overline{\mathcal{N}}}\right) \geq \overline{\mathscr{N}}^{\frac{1}{\alpha}}\left(\overline{\mathscr{N}}-\frac{2}{\bar{N}}\right)>0$. Thus, $\Psi(\hat{\alpha}, \mathscr{N})>0$.

Plugging the values of $\hat{A}$ and $\hat{b}$ into Eq. (2.1) we obtain

$x^{*}=\left(\frac{(\hat{A}-\mathscr{C}) \mathscr{N}}{\hat{b}(\mathscr{N}+\hat{\alpha})}\right)^{\frac{1}{\alpha}}=\mathscr{N} \mathscr{R}_{i}$ and $p^{*}=\frac{\hat{A} \hat{\alpha}+\mathscr{C} \mathscr{N}}{\mathscr{N}+\hat{\alpha}}$ $=\mathscr{P}$.
From the first inequality in Eq. (3.1) (with equality) 322 and the definition of $k$ it follows that

$$
\begin{aligned}
& \frac{\hat{\alpha}(\hat{A}-\mathscr{C})^{\frac{1+\hat{\alpha}}{\hat{\alpha}}} n^{\frac{1-\hat{\alpha}}{\hat{\alpha}}}}{\hat{b}^{\frac{1}{\hat{\alpha}}}(n+\hat{\alpha})^{\frac{1+\hat{\alpha}}{\hat{\alpha}}}}=\frac{\hat{\alpha}(\hat{A}-\mathscr{C})^{\frac{1+\hat{\alpha}}{\hat{\alpha}}} \mathscr{N}^{\frac{1-\hat{\alpha}}{\hat{\alpha}}}}{\hat{b}^{\frac{1}{\alpha}}(\mathscr{N}+\hat{\alpha})^{\frac{1+\hat{\alpha}}{\hat{\alpha}}}} \\
& \Leftrightarrow \frac{n^{\frac{1-\hat{\alpha}}{\hat{\alpha}}}}{(n+\hat{\alpha})^{\frac{1+\hat{\alpha}}{\hat{\alpha}}}}=\frac{\mathscr{N}^{\frac{1-\hat{\alpha}}{\hat{\alpha}}}}{(\mathscr{N}+\hat{\alpha})^{\frac{1+\hat{\alpha}}{\hat{\alpha}}},}
\end{aligned}
$$

which has $n=\mathscr{N}$ as a solution so the proof is 325 complete.

There are two main implications of this result. On the 327 one hand it points out the necessity of a good estimate of 328 $\alpha$ in order to judge the efficiency of a market. Notice that 329 first order conditions of profit maximization imply that 330 the elasticity of demand equals $\frac{\mathscr{N}(\mathscr{P}-\mathscr{C})}{\mathscr{P}}$ so neither the 331 elasticity of demand, nor price-marginal costs margins 332 are related to $\alpha$ and/or PWL. On the other hand, together 333 with the second part of iii'), it allows for markets 334 yielding PWL arbitrarily close to one, the main theo- 335 retical goal of this paper. The explanation of this, is that 336 we have constructed a market in which, in equilibrium, 337 profits are zero and, when tends to infinity, consumer 338 surplus is also zero since from Eq. (2.1) we have that 339

$U=\frac{\alpha}{(\alpha+1) b^{\frac{1}{\alpha}}}\left(\frac{n a}{n+a}\right)^{\frac{1+\alpha}{\alpha}}$, so $\lim _{\alpha \rightarrow \infty} \frac{\alpha}{(\alpha+1) b^{\frac{1}{\alpha}}}\left(\frac{n a}{n+\alpha}\right)^{\frac{1+\alpha}{\alpha}}=0 .{ }_{340}$

The intuition of the latter equation is that large values 342 of make inverse demand flatter and flatter so con- 343 sumer surplus goes to zero when $\alpha$ goes to infinity. The 344 difference with iii) in the previous section - where 345 $\lim _{\alpha \rightarrow \infty} L(\alpha, n)=0$ - arises from the fact that in the latter 346 industry profits are not zero, but when $\alpha$ tends to infinity 347 they tend to $\alpha$.

348

We now consider the case where fixed costs are 349 observable. In this case an observation is a list 350 $(\mathscr{P}, \mathscr{R} i, \mathscr{C}, \mathscr{N}, \mathscr{T})$ such that $\mathscr{T} \leq \mathscr{R}_{i}(\mathscr{P}-\mathscr{C})$ (i.e. profits 351 are non-negative). Consider the following condition that 352 guarantees that no firm will like to enter:

Definition 1. Observation $\left(\mathscr{P}, \mathscr{R}_{i}, \mathscr{C}, \mathscr{N}, \mathscr{T}\right)$ and $\alpha$ ful- 354 fill condition BE (Blockaded Entry) if

$$
\left(\frac{\mathscr{N}+\alpha+1}{\mathscr{N}+\alpha}\right)^{\frac{1+\alpha}{\alpha}}\left(\frac{\mathscr{N}}{\mathscr{N}+1}\right)^{\frac{1-\alpha}{\alpha}}>\frac{\mathscr{R}_{i}(\mathscr{P}-\mathscr{C})}{\mathscr{T}} .
$$

The right hand side can be interpreted as the rate of 358 (gross) profits. BE just says that the rate of profits 359 cannot be larger than a certain number which depends 360 on $\alpha$ and $\mathscr{N}$. The condition is more illuminating in 361 several special cases. For instance if $\alpha \rightarrow \infty$ condition 362 
$\mathrm{BE}$ reads $\mathscr{T}(\mathscr{N}+1)>\mathscr{N} \mathscr{R}_{i}(\mathscr{P}-\mathscr{C})$. When $\alpha \rightarrow$ - 1 condition BE reads $\mathscr{T}(\mathscr{N}+1)^{2}>\mathscr{N}^{2} \mathscr{R}_{i}(\mathscr{P}-\mathscr{C})$. Finally when $\alpha=1$, BE reads, $\mathscr{T}(\mathscr{N}+2)^{2}>$ $(\mathscr{N}+1)^{2} \mathscr{R}_{i}(\mathscr{P}-\mathscr{C})$.

Proposition 2. Given an observation $\left(\mathscr{P}, \mathscr{R}_{i}, \mathscr{C}, \mathscr{N}, \mathscr{T}\right)$ and a number $v$ such that $v=\operatorname{MI}(\hat{\alpha}, \mathscr{N}), \hat{\alpha} \in(-1,0) \cup$ $(0, \infty)$, if BE holds, there is a market $(\hat{A}, \mathscr{C}, \hat{b}, \hat{\alpha}, \hat{k})$ such that $\left(\mathscr{P}, \mathscr{X}_{i}, \mathscr{N}\right)$ is a Cournot equilibrium with free entry for this market (i.e. they fulfill Eqs. (2.1) and (3.1)), and $\mathrm{PWL} \geq v$.

Proof. (Virtually identical to the proof of Proposition 1). For $k$ equal to the minimum value in Eq. (3.1), PWL is given by Eq. (3.6). Choose $\hat{\alpha}$ such that $v=\operatorname{MI}(\hat{\alpha}, \mathscr{N})$. Set

$\hat{A}=\frac{\mathscr{P}(\mathscr{N}+\hat{\alpha})-\mathscr{C} \mathscr{N}}{\hat{\alpha}}, \hat{b}=\frac{(\hat{A}-\mathscr{C}) \mathscr{N}}{\mathscr{N}^{\hat{\alpha}} r_{i}^{\hat{\alpha}}(\mathscr{N}+\hat{\alpha})}$

This system can be solved, as we showed before. Plugging these values of $\hat{A}$ and $\hat{b}$ into Eq. (2.1) we obtain the required values of $x^{*}$ and $p^{*}$. Finally, the left hand side of the free entry condition Eq. (3.1) holds by the 381 definition of an observation. And when we plug the 382 values of $\hat{A}$ and $\hat{b}$ obtained above, the second inequality 383 of Eq. (3.1) reads

$\mathscr{T} \geq \frac{\mathscr{R}_{i}(\mathscr{P}-\mathscr{C})(\mathscr{N}+\hat{\alpha})^{\frac{1+\hat{\alpha}}{\hat{\alpha}}\left(\frac{\mathcal{N}+1}{\mathscr{N}}\right)^{\frac{1-\hat{\alpha}}{\hat{\alpha}}}}}{(\mathscr{N}+\hat{\alpha}+1)^{\frac{1+\hat{\alpha}}{\hat{\alpha}}}}$,

which under BE holds. When the above equation holds 386 with equality, $\mathrm{PWL}=\operatorname{MI}(\hat{\alpha}, \mathscr{N})=v$, so PWL $\geq v . \quad \square 387$

Comparing these with the results obtained in the 389 previous section we see that the consideration of fixed 390 costs allows the possibility of finding large PWL. This is 391 because in this case, we add the misallocation due to the 392 wrong number of firms to the misallocation due to the 393 wrong output. The former comes up to very large num- 394 bers because in our model the optimal number of firms is 395 one. ${ }^{9}$ But preferences play a role too: In the linear case, 396 values of PWL arbitrarily close to one cannot be obtained 397 for a given $n$. The reason is that the utility of the repre- 398 sentative consumer when $\alpha=1$ is always positive.

\section{Non-identical firms}

Suppose that firms have different $\operatorname{costs}$. Let $c_{i}$ be the marginal cost of firm $i$. Without loss of generality let $c_{1} \leq c_{i}$ for all $i$. Let $a_{i} \equiv A-c_{i}$. We will assume that for all $i,(n+\alpha-1) \alpha_{i}>\sum_{\mathbf{j} \neq i} a_{j}, b \sum_{j=1}^{n} a_{j}>0$ and $-A \alpha<\sum_{i=1}^{n} c_{i}$. These assumptions imply that, in equilibrium, all firms produce a positive output and market price is positive (see Eq. (4.1) below). Cournot equilibrium is easily shown to be unique and given by

$$
x_{i}^{*}=\frac{1}{\alpha}\left(\frac{\sum_{j=1}^{n} a_{j}}{b(n+\alpha)}\right)^{\frac{1}{\alpha}}\left(\frac{a_{i}(n+\alpha)}{\sum_{j=1}^{n} a_{j}}-1\right), x^{*}=\left(\frac{\sum_{j=1}^{n} a_{j}}{b(n+\alpha)}\right)^{\frac{1}{\alpha}} \text { and } p^{*}=\frac{A \alpha+\sum_{i=1}^{n} c_{i}}{n+\alpha} \text {. }
$$

Social welfare is $W=A x-b \frac{x^{x+1}}{\alpha+1}-\sum_{i=1}^{n} c_{i} x_{i}=\sum_{i=1}^{n} a_{i} x_{i}-b \frac{x^{\alpha+1}}{\alpha+1}$. In equilibrium,

$$
W^{*}=\frac{1}{\alpha} \sum_{i=1}^{n} a_{i}\left(\frac{\sum_{j=1}^{n} a_{j}}{b(n+\alpha)}\right)^{\frac{1}{\alpha}}\left(\frac{a_{i}(n+\alpha)}{\sum_{j=1}^{n} a_{j}}-1\right)-\frac{b}{\alpha+1}\left(\frac{\sum_{i=1}^{n} a_{i}}{b(n+\alpha)}\right)^{\frac{\alpha+1}{\alpha}},
$$

which when all $a_{i}$ 's are identical reduces to Eq. (2.3). In the optimal allocation only the technology in the hands of Firm 1 is used and accordingly

$$
x^{o}=\left(\frac{a_{1}}{b}\right)^{\frac{1}{\alpha}} \text { and } W^{o}=\frac{\alpha a_{1}^{\frac{\alpha+1}{\alpha}}}{(\alpha+1) b^{\frac{1}{\alpha}}} \text {. }
$$

\footnotetext{
${ }^{9}$ Overentry may also occur even if the marginal cost is increasing, see von Weizsäcker (1980), Mankiw and Whinston (1986) and Suzumura and Kiyono (1987).
} 
In order to have a workable expression for PWL that depends on observable variables alone, let us define $s_{i}$ as the market share of firm $i$. Clearly, and $\sum_{i=1}^{n} s_{i}=1$ and $s_{1} \geq s_{i}, i=2, \ldots, n$. Then, from Eq. (4.1),

$$
s_{i} \equiv \frac{x_{i}}{x}=\frac{a_{i}(n+\alpha)-\sum_{j=1}^{n} a_{j}}{\alpha \sum_{j=1}^{n} a_{j}} \Rightarrow a_{i}=\frac{\left(\alpha s_{i}+1\right) \sum_{j=1}^{n} a_{j}}{n+\alpha} .
$$

We will say that a list of market shares $\left(s_{1}, s_{2}, \ldots, s_{n}\right)$ is a Market Structure. It is clear from Eq. (4.4) that any vector $\left(a_{1}, a_{2}, \ldots a_{n}\right)$ yields a unique market structure compatible with Cournot equilibrium and that given a market structure we can construct a vector $\left(a_{1}, a_{2}, \ldots, a_{n}\right)$ (in fact an infinity number of vectors) whose Cournot equilibrium yields this market structure. Given this, we will focus on market structure that has the advantage of being observable.

Plugging the last part of Eq. (4.4) into Eq. (4.2) and after lengthy calculations we obtain PWL as a function of and the market structure, namely

$$
\mathrm{PWL}=\frac{\left(1+\alpha s_{1}\right)^{\frac{\alpha+1}{\alpha}}-(\alpha+1) \sum_{i=1}^{n} s_{i}^{2}-1}{\left(1+\alpha s_{1}\right)^{\frac{\alpha+1}{\alpha}}} \equiv P\left(s_{1}, \sum_{i=1}^{n} s_{i}^{2}, \alpha\right) .
$$

When all firms are identical, Eq. (4.5) reduces to Eq. (2.4). It is noteworthy that PWL here depends only on three variables:

$-\alpha$.

- The market share of the largest firm $s_{1}$.

- The Hirschman-Herfindahl index of concentration denoted by $H \equiv \sum_{i=1}^{n} s_{i}^{2} \cdot{ }^{10}$

Eq. (4.5) allows computation of PWL from $s_{1}$ and $H$ assuming that demand is linear or isoelastic (where $\alpha$ is the inverse elasticity of demand). It also allows to plot PWL as a function of $\alpha$ for actual market structures and see what this function looks like, see Corchón (2006) for a simple application to the Spanish gasoline market.

Notice the following properties of $P()$ as defined by Eq. (4.5): ${ }^{11}$

i") $\lim _{\alpha \rightarrow-1} \mathrm{P}\left(s_{1}, H, \alpha\right)=0$.

ii") $\lim _{\alpha \rightarrow \infty} P\left(s_{1}, H, \alpha\right)=\frac{1}{s_{1}}\left(s_{1}-\sum_{i=1}^{n} s_{i}^{2}\right)$.

iii") $P(\cdot, H, \alpha)$ is increasing on $s_{1}$.

iv") $P\left(\mathrm{~s}_{1}, \cdot, \alpha\right)$ is decreasing on $H$.

v") $\lim _{\alpha \rightarrow 0} \operatorname{PWL}\left(s_{1}, H, \alpha\right)=\frac{e^{s_{1}}-1-H}{e^{s_{1}}}$

i") is identical to i). When firms are identical ii") reduces to ii). Point iii') agrees with the received wisdom: the larger the dominant firm, the closer to monopoly, and hence the larger the PWL is. However, iv") is counterintuitive because it says the larger the concentration, the lower the WL. The reason is that when $H$ increases, production is shifted to the less efficient firms which causes social welfare to fall. Finally v") allows us to extend $P\left(\mathrm{~s}_{1}, H, \cdot\right)$ to $\alpha=0$ preserving continuity.

We now discuss why the approach followed in the previous section will not work here. An Observation is a list $\left(\mathscr{P}, \mathscr{R}_{\mathbb{R}}, \ldots, \mathscr{R}_{\mathcal{N}}, \mathscr{C}_{\mathbb{R}}, \ldots, \mathscr{C}_{\mathcal{N}}\right)$ where $\mathscr{P}$ is market price and $\mathscr{R}_{i}$ and $\mathscr{C}_{i}(<\mathscr{P})$ are the output and the marginal cost of firm $i$. A Market is a list $\left(\mathrm{A}, c_{1}, \ldots, c_{n}, b, \alpha\right)$ such that $(n+\alpha-1) a_{i}>\sum_{j \neq \mathrm{i}} a_{j}, \alpha>-1, b \sum_{j=1}{ }^{n} a_{j}>0, b \alpha>0$, and $-A \alpha<\sum_{i=1}^{n} c_{i}$. Clearly, not all observations are compatible with the model. In particular, the number of variables in an observation is $2 n+1$ and the number of parameters defining a market is $n+3$. With $n>2$, the number of parameters will be, in general, unable to generate the required observations. Also, first order conditions of profit maximization imply that

$$
\frac{\mathscr{R}_{\mathscr{D}}}{\mathscr{R}_{\mathscr{J}}}=\frac{\mathscr{P}-\mathscr{C}_{\mathscr{I}}}{\mathscr{P}-\mathscr{C}_{\mathscr{g}}} .
$$

This relation may fail even for the case $n=2$. Given this, we will study how PWL depends on $\alpha, n$ and the market structure focussing our attention on limiting cases, i.e. when PWL is maximal or minimal. Our first result is that when

\footnotetext{
$\overline{{ }^{10} \text { In fact, }} s_{1}$ and $H$ are not independent but we prefer to write Eq. (4.5) in this way to highlight the role of $H$ in the formula.

${ }^{11}$ As we mentioned before, we take $s_{1}$ and $H$ as independent when in fact they are not.
} 
$\alpha, n$, and the market structure can be chosen simultaneously, PWL can be arbitrarily close to one and at the same time the concentration index $H$ arbitrarily low.

Proposition 3. There exists $\left(\alpha, n, s_{1}, \ldots, s_{n}\right)$ for which $P W L$ is arbitrarily close to one and $H$ is arbitrarily close to zero.

Proof. From iv") the maximal PWL occurs when $s_{2}=s_{3}=, \ldots,=s_{n}$. Denoting these shares by $y$, we have that $s_{1}+(n-1)$ $y=1$. Plugging this in Eq. (4.5) we have that

$$
P\left(s_{1}, n, \alpha\right) \equiv \frac{\left(1+\alpha s_{1}\right)^{\frac{\alpha+1}{\alpha}}-(\alpha+1)\left(s_{1}^{2}+\frac{\left(1-s_{1}\right)^{2}}{n-1}\right)-1}{\left(1+\alpha s_{1}\right)^{\frac{\alpha+1}{\alpha}}} .
$$

PWL is increasing on $n$ so the maximum PWL obtains when $n$ is arbitrarily large, i.e.

$$
\lim _{n \rightarrow \infty} P\left(s_{1}, n, \alpha\right)=\frac{\left(1+\alpha s_{1}\right)^{\frac{\alpha+1}{\alpha}}-(\alpha+1) s_{1}^{2}-1}{\left(1+\alpha s_{1}\right)^{\frac{\alpha+1}{\alpha}}} .
$$

We easily compute $\lim _{\alpha \rightarrow \infty} \lim _{n \rightarrow \infty} P\left(s_{1}, n, \alpha\right)=\lim _{n \rightarrow \infty} \lim _{\alpha \rightarrow \infty} P\left(s_{1}, n, \alpha\right)=1-s_{1}$. Thus when $\alpha$ and $n$ are very large and $s_{1}$ very small, PWL is arbitrarily close to one (since limits are interchangeable our procedure is robust). The restriction $s_{1} \geq s_{i}, i=2, \ldots, n$ when firms $2, \ldots, n$ are identical, is equivalent to $n s_{1} \geq 1$. This inequality holds when the order of magnitude at which $n$ tends to $\infty$ is larger than the order of magnitude at which $s_{1}$ tends to 0 .

Finally, it can be easily shown that when firms 2 to $n$ are identical,

$$
H=\frac{n s_{1}^{2}+1-2 s_{1}}{n-1}=\frac{s_{1}^{2}+\frac{1}{n}-2 \frac{s_{1}}{n}}{1-\frac{1}{n}}
$$

which when $n \rightarrow \infty$ and $s_{1} \rightarrow 0$ tend to zero.

From the previous proof it follows that for $n$ and $\alpha$ large, $P W L \simeq 1-\sqrt{H}$ which highlights the point made before about the relationship between concentration and WL.

It can be shown that if one of the variables in our construction is held fixed, can be made large, but not close to one, and is again far from being a reliable measure of Corchón (2006), pp. 19-21. We now perform a more demanding exercise where PWL is studied by varying only one variable, either the market structure or $\alpha$.

We first concentrate on how market shares affect PWL. A market structure such that $s_{1}>s_{2}=, \ldots,=s_{n}>0$ will be called a Dominant Firm. A limit case of a dominant firm is Monopoly where only $s_{1}$ is positive.

Proposition 4. For $\alpha>0$, PWL is maximized when the market structure is a dominant firm with $s_{1}=\frac{n+3}{2 n+2}$ if $\alpha=$ 1 and $s_{1}=\frac{-n-1+\sqrt{1+\alpha n+\alpha^{2} n+\alpha n^{2}}}{\alpha n-n}$ if $\alpha \neq 1$. For $\alpha<0$ the market structure that maximizes PWL is monopoly.

Proof. The maximum of PWL in Eq. (4.5) over $\sum_{i=1}^{n} s_{i}=1$ exists (by Weierestrass' theorem). As mentioned before, it occurs when $s_{2}=s_{3}=, \ldots,=s_{n}$. So, let us consider PWL as given by Eq. (4.6). The extrema of this expression with respect to $s_{1}$ can be located, either when $\frac{\partial P\left(s_{1}, n, \alpha\right)}{\partial s_{1}}=0$ or in the bounds of the interval in which $s_{1}$ must lie, namely $s_{j} \leq s_{i} \leq 1$ for all $j>1$. Since $(n-1) s_{j} \leq s_{1}$ the previous inequality can be written as $\frac{1}{n} \leq s_{1} \leq 1$. Now, rewrite Eq. (4.6) as follows:

$$
P\left(s_{1}, n, \alpha\right)=1-\frac{(\alpha+1)\left(n s_{1}^{2}-2 s_{1}+1\right)+n-1}{(n-1)\left(1+\alpha s_{1}\right)^{\frac{\alpha+1}{\alpha}}} .
$$

$$
\frac{\partial P}{\partial s_{1}}=\frac{s_{1}^{2}\left(n-n \alpha^{2}\right)-s_{1}(2+2 n+2 \alpha+2 n \alpha)+2+\alpha(3+n+\alpha)+n}{(n-1)\left(\alpha s_{1}+1\right)^{\frac{1}{\alpha}+2}}
$$

$$
\frac{\partial P}{\partial s_{1}}=0 \Leftrightarrow s_{1}^{2}\left(n-n \alpha^{2}\right)=2 s_{1}(1+n+\alpha+n \alpha)-2-\alpha(3+n+\alpha)-n
$$


We have three possible cases: If $\alpha=1$, the solution to Eq. (4.9) is $s_{1}^{*}=\frac{n+3}{2 n+2} \in\left[\frac{1}{n}, n\right]$. Then, the maximum must be located either at $s_{1}=\frac{1}{n}$, at $s_{1}=1$ or at $s_{1}=\frac{n+3}{2 n+2}$. We easily compute,

$$
P(1, n, 1)=\frac{1}{4}, P\left(\frac{1}{n}, n, 1\right)=\frac{1}{(n+1)^{2}}, P\left(\frac{n+3}{2 n+2}, n, 1\right)=\frac{n+1}{3 n+5} .
$$

From these expressions we obtain the desired result.

If $\alpha>1$ from the first order condition we obtain two solutions,

$$
s_{1}^{*}=\frac{-n-1 \pm \sqrt{1+\alpha n+\alpha^{2} n+\alpha n^{2}}}{\alpha n-n} .
$$

Clearly only the solution with a plus sign in front of the square root is feasible. We will show that for this solution $s_{1}^{*} \in\left[\frac{1}{n}, 1\right]$. If $\frac{1}{n}>s_{1}^{*}$ we would have $\alpha^{2}(n-1)+n^{2}(\alpha-1)-\alpha n+1<0$ which is impossible because the left hand side achieves a minimum when $n=2$ and $\alpha=1$. Similarly, if $s_{1}^{*}>1, \alpha n-\alpha-n+1<0$, which again is impossible.

Finally, notice that since there is only one value of $s_{1}$ for which $\frac{\partial P\left(s_{1}, n, \alpha\right)}{\partial s_{1}}=0$ the shape of $P(\cdot, n, \alpha)$ is determined by the sign of $\frac{\partial P\left(s_{1}, n, \alpha\right)}{\partial s_{1}}$ at $s_{1}=\frac{1}{n}$ and $s_{1}=1$. From Eq. (4.8),

$$
\operatorname{sign} \frac{\partial P\left(\frac{1}{n}, n, \alpha\right)}{\partial s_{1}}=\operatorname{sign}\left(n+\alpha+n \alpha-\frac{1}{n}+\alpha^{2}-\frac{2}{n} \alpha-\frac{1}{n} \alpha^{2}\right)
$$

which is positive because the expression on the right hand side is increasing in $\alpha$ and for $\alpha=-1$ equals to zero. Also from Eq. (4.8) we obtain that

$$
\operatorname{sign} \frac{\partial P(1, n, \alpha)}{\partial s_{1}}=\operatorname{sign}\left(\alpha-n \alpha+\alpha^{2}-n \alpha^{2}\right)=\operatorname{sign}(\alpha(1+\alpha)(1-n))
$$

which is negative so the interior solution is indeed a maximum.

Finally let us consider the case $\alpha<1$. Suppose that the negative root in Eq. (4.10) is less than one. Then

$$
\frac{-n-1-\sqrt{1+\alpha n+\alpha^{2} n+\alpha n^{2}}}{\alpha n-n}<1 \Leftrightarrow-\sqrt{1+\alpha n+\alpha^{2} n+\alpha n^{2}}>\alpha n+1,
$$

which is impossible. So there is, at most, one interior solution. Suppose first that $\alpha>0$. From Eqs. (4.11)-(4.12) we get that $\operatorname{sign} \frac{\partial P\left(\frac{1}{n}, n\right)}{\partial s_{1}}$ is positive and sign $\frac{\partial P(1, n, \alpha)}{\partial s_{1}}$ is negative which implies that maximum PWL is achieved at the interior solution. If $\alpha=0$ the positive root in Eq. (4.10) equals one. Finally, if $\alpha<0$, from Eqs. (4.11)-(4.12), we have that $\operatorname{sign} \frac{\partial P\left(\frac{1}{n}, n, \alpha\right)}{\partial s_{1}}$ and $\operatorname{sign} \frac{\partial P(1, n, \alpha)}{\partial s_{1}}$ are both positive which given that there is, at most one value of $s_{1}$ for which sign $\frac{\partial P(\cdot, n, \alpha)}{\partial s_{1}}$ switches from positive to negative means that $P(\cdot, n, \alpha)$ is increasing, so it achieves the maximum when $s_{1}=1$.

Proposition 4 says that the most deleterious market structure is not always monopoly, the target of the wrath of economists since Adam Smith. In many cases a dominant firm structure is worse because firms other than do not add much competition to the market and they are technologically inefficient. We notice that under maximal PWL,

$$
H=\frac{n s_{1}^{2}+1-2 s_{1}}{n-1} \text { and PWL }=\frac{\left(1+\alpha s_{1}\right)^{\frac{\alpha+1}{\alpha}}-(\alpha+1)\left(n s_{1}^{2}+\frac{\left(1-s_{1}\right)^{2}}{n-1}\right)-1}{\left(1+\alpha s_{1}\right)^{\frac{\alpha+1}{\alpha}}},
$$

so $H$ decreases with $n$ but PWL increases with $n$. And $H$ increases with $s_{1}$ but PWL not necessarily so. Thus, again, the concentration index $H$ is a poor measure of WL.

The maximum PWL for given $n$ and $\alpha$ is obtained by plugging the value of $s_{1}$ that maximizes PWL as found in Proposition 4 and denoted by $s(\alpha, n)$, into $P\left(s_{1}, n, \alpha\right)$. Let $P(s(\alpha, n), n, \alpha) \equiv F(\alpha, n)$, say. 
It can be shown that $F(\alpha, \cdot)$ is increasing in $n$ which implies that, for any number of firms, it is possible to find the PWL of, at least, $F(\alpha, 2)$ which for values of $\alpha \in(0,50]$ never goes below. Finally, we state two limiting properties of $F(\cdot, \cdot)$ :

$$
\begin{aligned}
\lim _{\alpha \rightarrow \infty} F(\alpha, n) & =\frac{(\sqrt{n})^{3}+\sqrt{n}-2 n}{(\sqrt{n})^{3}-\sqrt{n}} \\
\lim _{n \rightarrow \infty} F(\alpha, n) & =1-\frac{(\sqrt{\alpha}-1)^{2}+(\alpha+1)+(\alpha-1)^{2}}{(\alpha-1)^{\frac{\alpha-1}{\alpha}}(\alpha \sqrt{\alpha}-1)^{\frac{\alpha+1}{\alpha}}}
\end{aligned}
$$

Notice that in both cases PWL is high even for small values of $\alpha$ and $n$. It is clear that $\lim _{n \rightarrow \infty}, \alpha \rightarrow \infty F(\alpha, n)=$ $\lim _{\alpha \rightarrow \infty}, n \rightarrow \infty F(\alpha, n)=1$.

We now turn to the study of the market structure that minimizes PWL.

Lemma 1. Suppose that $\left(\hat{s}_{1}, \hat{s}_{2}, \ldots, \hat{s}_{n}\right)$ minimizes $P\left(s_{1}, \sum_{i=1}^{n} s_{i}^{2}, \alpha\right)$. Then $\nexists \hat{s}_{i}, \hat{s}_{j}, j>1$ such that $\hat{s}_{1}>\hat{s}_{i} \geq \hat{s}_{j}>0$.

Proof. Increasing $\hat{s}_{i}$ by a small amount, say $\mathrm{d} x$, and decreasing $\hat{s}_{j}$ by $\mathrm{d} x$ too is feasible - i.e. $\hat{s}_{i}+\mathrm{dx}$ and $\hat{s}_{j}-d x \in\left[0, s_{1}\right]$ increases $H$ and so decreases PWL which contradicts that is minimized.

Lemma 1 implies that only three market structures might minimize PWL: 1) All firms produce the same output 2) All firms minus one, say $n$, produce the same output. 3) A number of firms, say $1, \ldots, m$ with $m<n$ produce the same output, and the remaining firms produce zero output. But the last option cannot minimize PWL since it was established that when all firms are identical, PWL decreases with the number of (active) firms (Property iv) in Section 2). So we are left with options 1 and 2.

\section{Proposition 5. The market structure that minimizes PWL is when all firms produce the same output.}

Proof. Notice that market structures 1 and 2 can be written as $(x, x, \ldots, 1-(n-1) x)$ with $x \in\left[\frac{1}{n-1}, \frac{1}{n}\right]$, where the lower bound of this interval comes from $1 \geq(n-1) x$. In this case $H=(n-1) x^{2}+(1-(n-1) x)^{2}$. Plugging $H$ into Eq. (4.5) we obtain

$$
\mathrm{PWL}=1-\frac{(\alpha+1)\left((n-1) x^{2}+(1-(n-1) x)^{2}\right)+1}{(1+\alpha x)^{\frac{\alpha+1}{\alpha}}} \equiv \mathrm{PW}(\alpha, x, n) .
$$

$$
(1+\alpha x)^{\frac{\alpha+1}{\alpha}}
$$

Now, computing $\frac{\partial \mathrm{PW}(\alpha, x, n)}{\partial x}$ this expression is found to be equal to

$$
\frac{-(1+\alpha)}{(1+x \alpha)^{\frac{1+\alpha}{\alpha}}}\left[2 n^{2} x-2 n x-2 n+2-\frac{(1+\alpha)\left((n-1) x^{2}+(1-(n-1) x)^{2}\right)+1}{1+x \alpha}\right]
$$

Solving for $\frac{\partial P W(\alpha, x, n)}{\partial x}=0$ we obtain the following. If $\alpha=1$,

$$
\frac{\partial \mathrm{PW}(\alpha, x, n)}{\partial x}=0 \Leftrightarrow 4 n+4 x+2-4 n^{2} x=0 \Leftrightarrow x=\frac{2 n+1}{2 n^{2}-2}<\frac{1}{n-1} .
$$

So only boundary solutions are feasible and PWL is minimized when $x=\frac{1}{n}$. If $\alpha \neq 1$,

$$
\frac{\partial \mathrm{PW}(\alpha, x, n)}{\partial x}=0 \Leftrightarrow x=\frac{-n^{2}+1 \pm \sqrt{n^{4}+1+2 \alpha n^{3}+\alpha^{2} n^{2}-3 \alpha n^{2}-\alpha^{2} n-2 n^{3}+\alpha n}}{(\alpha-1)\left(n^{2}-n\right)} .
$$

Suppose that $\alpha>1$. Clearly, the negative root is not feasible, so consider the positive root, say $x^{*}$. If $x^{*} \leq \frac{1}{n}$, it must be that $(n-1)\left(\alpha^{2}+\alpha n-1-n\right) \leq 0$ which for $n>2$ and $\alpha>1$ is impossible.

Suppose that $\alpha<1$. If the negative root is less than or equal to $\frac{1}{n}$, we have that $-\sqrt{n^{4}+1+2 \alpha n^{3}+\alpha^{2} n^{2}-3 \alpha n^{2}-\alpha^{2} n-2 n^{3}+\alpha n} \geq$ $(n+\alpha)(n-1)$ which is impossible. Take the positive root. If this root is larger than or equal to $\frac{1}{n-1}$, then $n(1-\alpha) \leq \alpha^{2}-$ 
$3 \alpha+2$ or $n \leq \frac{\alpha^{2}-3 \alpha+2}{1-\alpha}$. The right hand side of this inequality has a maximum at 3 when $\alpha \rightarrow-1$ Since this value of is never actually achieved, this inequality only may hold when $n=2$. But $\frac{\partial \mathrm{PW}(\alpha, 0.5,2)}{\partial x}=\frac{0.5 \alpha+1.5}{0.5 \alpha+1}>0$ which means that the minimum is achieved at the boundaries of $x$. Since in this case these bounds imply monopoly and duopoly, by iv) in Section 2 we achieve the desired result.

An implication of Proposition 5 is that disregarding firms heterogeneity stacks the deck in favour of small WL. Also, minimal PWL is given by the function $L(\cdot, \cdot)$ in Eq. (2.4). Recall that maximal PWL is given by the function $F(\alpha, \cdot)$ (defined in the second paragraph after the end of Proposition 4). Notice that since $L(\alpha, \cdot)$ is decreasing in $\alpha$ and $F(\alpha, \cdot)$ is increasing in $n$, the difference between maximal and minimal PWL increases with $n$ for a given $\alpha$. Also, since $P(\cdot, n, \alpha)$ is continuous in $s_{1}$, any PWL between $L(\alpha, n)$ and $F(\alpha, n)$ is reachable by the choice of $s_{1}$.

Finally we consider the effect of $\alpha$ alone on PWL. We have little to say about the value of $\alpha$ that maximizes PWL because first order condition of maximization with respect to $\alpha$ is not very informative. However, the continuity of $P\left(s_{1}, n, \cdot\right)$ has an interesting implication. Let $V \equiv \max \left\{\frac{s_{1}-H}{s_{1}}, \frac{\left(1+s_{1}\right)^{2}-2 H-1}{\left(1+s_{1}\right)^{2}}, \frac{e^{s_{1}}-1-H}{e^{s_{1}}}\right\}$. The values in the bracket are respectively, $P\left(s_{1}, n, 0\right), P\left(s_{1}, n, 1\right)$ and $\lim _{\alpha \rightarrow \infty} P\left(s_{1}, n, \alpha\right)$. Then, we have:

\section{Corollary 1. Any $P W L \in(0, V)$ is obtainable by the choice of $\alpha$.}

\section{Final comments}

When one observes public policies on oligopolies one sees some concern about the number and the relative size of firms. But the question of the output set by oligopolists is cause of little or no concern at all. This paper provides some justification to this attitude: We found that WL due to the divergence between equilibrium and optimal output are small, even with as few as four firms in the market as shown in Section 2. On the contrary WL due to the number and relative size of firms can be quite substantive as found in Sections 3 and 4. This conclusion, though, is likely to be exaggerated by our assumption that the optimal number of firms is one. Other important factors are the consideration of product differentiation and other solution concepts, e.g. Bertrand or Stackelberg equilibria, see Cable et al. (1994) for the case of duopoly and quadratic utility. In fact, two of the main conclusions of Cable et al. (1994, p. 98) are that "the particular form of oligopolistic interaction exerts a major influence on the level of welfare" and "the power of inter-firm rivalry to further social welfare is highly sensitive to the degree of product differentiation in he market" (pp. 98-9). Moreover, in a dynamic framework WL can be larger than here because firms may collude. Thus, our results are just a first cut to the problem.

Our results have a number of implications for the applied literature.

1. To measure WL due to oligopolistic output setting may be misguided because these losses are likely to be small. However WL due to overentry or to asymmetric firms can be quite substantial. Lack of consideration of these points biases downwards our estimates of WL.
2. Bresnahan and Reiss (1991) found markets where, as 616 the number of firms increased beyond three, the ${ }_{617}$ competitive effect of additional firms on average ${ }_{618}$ markups was exhausted, a fact that suggests that the ${ }_{619}$ outcome is very close to perfect competition. A 620 possible explanation for their findings is that they ${ }_{621}$ considered markets where asymmetries and econo- 622 mies of scale were possibly small (i.e. doctors, ${ }_{623}$ dentists, druggists, plumbers and tire dealers). In ${ }_{624}$ contrast, Campbell and Hopenhayn (2002) find that ${ }_{625}$ this competitive effect persists with a large number ${ }_{626}$ of firms in markets were firms are asymmetric (and 627 the product is differentiated). Our findings in this ${ }_{628}$ paper may help to understand the difference in ${ }_{629}$ results in these two papers.

3. The impact of mergers and collusive agreements on 631 social welfare depends on the characteristics of the ${ }_{632}$ market. For instance, with identical firms and no ${ }_{633}$ fixed costs our results in Section 2 suggest that anti- ${ }_{634}$ trust authorities should not be very concerned with ${ }_{635}$ mergers that do not bring the number of competing ${ }_{636}$ firms below, say four. However merging from ${ }_{637}$ duopoly to monopoly approximately doubles PWL. ${ }_{638}$ If firms are not identical or there are fixed costs, ${ }_{639}$ traditional measures of concentration fail to capture ${ }_{640}$ the full size of WL.

4. WL depend crucially on the parameter that cannot be ${ }_{642}^{641}$ observed, but can be estimated. Our results point out ${ }_{643}$ the importance of the estimation of for the proper ${ }_{644}$ account of WL. This may be problematic because to ${ }_{645}$ say something empirical about the local (around the ${ }_{646}$ actual price) characteristics of the demand curve ${ }_{647}$ sounds reasonable, but our approach requires global 648 information about those characteristics. 


\section{References}

Anderson, S.P., Renault, R., 2003. Efficiency and surplus bounds in Cournot competition. Journal of Economic Theory 113, 253-264.

Bresnahan, T., Reiss, P., 1991. Entry and competition in concentrated markets. Journal of Political Economy 99 (5), 977-1009.

Cable, J., Carruth, A., Dixit, A., 1994. Oligopoly and welfare. In: Cable, J. (Ed.), Current Issues in Industrial Economics. Macmillan Press, London.

Campbell, J., Hopenhayn, H., 2002. Market size matters. NBER Working Paper 9113.

Corchón, L., 2006. Welfare losses under Cournot competition. Working paper 06-39, Universidad Carlos III, June.

Cournot, A.A., 1838. Recherches sur les principles mathematiques de la thèory des Richesses. Hachette, Paris.

Dasgupta, P., Ushio, Y., 1981. On the rate of convergence of oligopoly equilibria in large markets: an example. Economics Letters 8, 13-17.

Daughety, A., 1990. Beneficial concentration. American Economic Review 80 (5), 1231-1237.

Farrell, J., Shapiro, C., 1990. Horizontal merger: an equilibrium analysis. American Economic Review 80 (1), 107-126.

Fraysse, J., Moreaux, M., 1981. Cournot equilibrium in large markets under increasing returns. Economics Letters 8, 217-220.

Guesnerie, R., Hart, O., 1985. Welfare losses due to imperfect competition. International Economic Review 26 (2), 525-545.

Harberger, A.C., 1954. Monopoly and resource allocation. American Economic Review: Papers and Proceedings 44, 77-87.

Hotelling, H., 1938. The general welfare in relation to problems of taxation and of railways and utility rates. Econometrica 6, $242-269$.
Johari, R., Tsitsiklis, J. (2005). Efficiency loss in Cournot games. 680 Mimeo, January 28, 2005.

Lahiri, S., Ono, Y., 1988. Helping minor firms reduces welfare. 682 Economic Journal 1199-1203 December. 683

Leibenstein, H., 1966. Allocative efficiency versus x-efficiency. 684 American Economic Review 56, 392-425.

López-Cuñat, J.M., 1999. One-stage and two-stage entry Cournot 686 equilibria. Investigaciones Económicas 23 (1), 115-128. 687

Mankiw, G., Whinston, M., 1986. Free entry and social inefficiency. 688 Rand Journal of Economics 17 (1), 48-58. 689

McHardy, J.P., 2000. Miscalculations of monopoly and oligopoly 690 welfare losses with linear demand. Hull Economic Research 691 Papers, November.

Novshek, W., 1980. Cournot equilibrium with free entry. Review of 693 Economic Studies 42, 473-486. 694

Suzumura, K., Kiyono, K., 1987. Entry barriers and economic welfare. 695 Review of Economic Studies 54, 157-167. 696

Tullock, G., 1967. The welfare costs of tariffs, monopolies, and theft. 697 Western Economic Journal 5, 224-232.

698

Tullock, G., 2003. The origin rent-seeking concept. International 699 Journal of Business and Economics 2 (1), 1-8.

Ushio, Y., 1983. Cournot equilibria with free entry: the case of 701 decreasing average cost function. Review of Economic Studies 50, 702 347-354.

von Weizsäcker, C.C., 1980. A welfare analysis of barriers to entry. 704 Bell Journal of Economics 11, 399-420.

705

Yarrow, G.K., 1985. Welfare losses in oligopoly and monopolistic 706 competition. Journal of Industrial Economics 33 (4), 515-529. 707 